\title{
Synergisic effect of APRIL knockdown and Jiedu Xiaozheng Yin, a Chinese medicinal recipe, on the inhibition of hepatocellular carcinoma cell proliferation
}

\author{
ZHIZHEN LIU ${ }^{1}$, LIANMING LIAO ${ }^{2,3}$, ZHIYUN CAO $^{2}$, XUZHENG CHEN $^{2}$ and JIAN DU ${ }^{1,2}$ \\ ${ }^{1}$ College of Integrative Medicine and ${ }^{2}$ Academy of Integrative Medicine, Fujian University of Traditional Chinese Medicine; \\ ${ }^{3}$ Laboratory Center, Union Hospital of Fujian Medical University, Fuzhou, Fujian 350122, P.R. China
}

Received June 30, 2016; Accepted September 1, 2016

DOI: $10.3892 /$ or.2016.5339

\begin{abstract}
It is well documented that A proliferation-inducing ligand (APRIL), a member of the tumor necrosis factor superfamily, plays a crucial role in the occurrence and development of tumors. In the present study, we evaluated the synergistic effect of APRIL knockdown and Jiedu Xiaozheng Yin (JXY), a Traditional Chinese Medicinal recipe, on the inhibition of hepatocellular carcinoma (HCC) cell proliferation and elucidated the underlying mechanism. The results demonstrated that both APRIL knockdown using small interfering RNA (siRNA) and JXY treatment could trigger cell cycle arrest and cell apoptosis, and suppress HCC cell proliferation through an $\mathrm{NF}-\kappa \mathrm{B}-$ related pathway. Synergism was further demonstrated between APRIL knockdown and JXY treatment. In conclusion, these results indicate that APRIL is a target gene for HCC and combination of siRNA-APRIL and JXY application holds great promise as a novel approach for the treatment of APRIL-positive HCC.
\end{abstract}

Correspondence to: Professor Jian Du, College of Integrative Medicine, Fujian University of Traditional Chinese Medicine, 1 Qiuyang Road, Shangjie, Minhou, Fuzhou, Fujian 350122, P.R. China

E-mail: 1325370721@qq.com

Abbreviations: HCC, hepatocellular carcinoma; APRIL, A proliferation-inducing ligand; JXY, Jiedu Xiaozheng Yin; $\mathrm{NF}-\kappa \mathrm{B}$, nuclear factor- $\kappa \mathrm{B}$; VEGF, vascular endothelial growth factor; HDW, Hedyotis diffusa Willd; SF, Sophora flavescens; PC, Pseudobulbus cremastrae; TNF, tumor necrosis factor; TNFSF13, tumor necrosis factor superfamily 13; DMEM, Dulbecco's modified Eagle's medium; FBS, fetal bovine serum; GAPDH, glyceraldehyde 3-phosphate dehydrogenase; RIPA, radioimmunoprecipitation; MTT, methyl thiazolyl tetrazolium; ECL, electrochemiluminescence; TUNEL, terminal deoxynucleotidyl transferase dUTP nick end labeling; ANOVA, analysis of variance

Key words: A proliferation-inducing ligand, Jiedu Xiaozheng Yin, hepatocellular carcinoma, nuclear factor- $\mathrm{kB}$

\section{Introduction}

Hepatocellular carcinoma (HCC), one of the most common malignancies, represents the third leading cause of cancer-related deaths worldwide (1). Although screening in high-risk populations has increased the detection of early-stage HCC and consequently improved survival, most cases still present with advanced and unresectable stages due to intrahepatic metastasis and vascular invasion $(2,3)$. Seeking alternative therapies to improve the survival of HCC patients has been an urgent task for oncologists.

A proliferation-inducing ligand (APRIL), also known as TALL-2 and TNFSF13, was identified via database mining in 1998 by Hahne et al (4). APRIL is a member of the TNF family located on human chromosome 17p13. APRIL expression is low in normal cells, including monocytes, dendritic and $\mathrm{T}$ cells $(5,6)$. However, studies have demonstrated that it is overexpressed in many tumors, which suggest that APRIL plays a crucial role in the occurrence and development of these tumors (7-9).

Heat-clearing and detoxifying Chinese herbs, which play an important role in antitumor therapy in China, involve the use of extracts from these herbs for the treatment of different types of cancer (10-12). Jiedu Xiaozheng Yin (JXY), a polyherbal Traditional Chinese Medicine (TCM) recipe, is composed of Hedyotis diffusa Willd (HDW), Sophora flavescens (SF), Prunella and Pseudobulbus Cremastrae (PC) and is used as a heat-clearing and detoxicating adjuvant therapy for HCC. Our previous study demonstrated that JXY can inhibit the angiogenesis of tumors via the downregulation of vascular endothelial growth factor-A (VEGF-A) and vascular endothelial growth factor receptor-2 (VEGFR-2) expression (13). Moreover, an ethyl acetate extract from JXY inhibits the cell proliferation of HCC by suppressing polycomb gene product Bmil and Wnt/ $\beta$-catenin signaling $(14,15)$. A randomized control trial showed that the addition of JXY to the standard treatment of stage III HCC patients could improve the immune function of patients, decrease recurrence and increase overall survival (16).

In the present study, we evaluated the synergistic effect of APRIL knockdown and JXY treatment on the proliferation of HCC cells and elucidated the underlying mechanism. 


\section{Materials and methods}

Preparation of the herbal medicine. JXY is composed of HDW (30 g), Prunella (15 g), PC (15 g) and SF (15 g). These four herbs of JXY were purchased from the Guo Yi Tang Hospital of Fujian University of Traditional Chinese Medicine (Fuzhou, China). The quality of the 4 medicinal plants met the criterion of the Pharmacopoeia of the People's Republic of China. To prepare the crude water extract, 300, 150, 150 and $150 \mathrm{~g}$ of the 4 medicinal plants, HDW, Prunella, PC and $\mathrm{SF}$, respectively, were crushed into powders. Then, they were mixed together and immersed in 101 of distilled water. The mixture was simmered for $2 \mathrm{~h}$ and filtered. Subsequently, the solution was concentrated by a rotary evaporator and stored at $4^{\circ} \mathrm{C}$ until use.

Animals. The clean grade level male Sprague-Dawley (SD) rats (licence no. SCXK (Hu) 2007-0005), with a body weight of 180-220 g, were purchased from Shanghai SLAC Experimental Animal Co., Ltd. (Shanghai, China). All rats were maintained under a regulated temperature $\left(18-22^{\circ} \mathrm{C}\right)$ and a relative constant humidity (50-60\%). All animal handling and experimental procedures were approved by the Animal Care and Use Committee of Fujian University of TCM.

Preparation of JXY-containing serum. The 20 SD rats were divided into 4 groups using a random digit table method ( $n=10 /$ group) and were administered JXY solution orally at a dose of $9 \mathrm{~g} / \mathrm{kg} /$ day or saline respectively twice a day for 7 days. One hour after the last treatment, blood was collected from the main ventral artery under aseptic conditions, and centrifuged at 3,000 rpm for $15 \mathrm{~min}$. Serum from the 2 groups was referred to as JXY and control serums, respectively. All sera were filtered through a $0.22-\mu \mathrm{m}$ filter membrane and inactivated at $56^{\circ} \mathrm{C}$ water for $30 \mathrm{~min}$ and stored at $-20^{\circ} \mathrm{C}$ until its use in pharmacological studies.

Cell culture. The HepG2 HCC cell line was purchased from the Cell Bank of the Shanghai Institute of Biochemistry and Cell Biology, Chinese Academy of Sciences (Shanghai, China), and cultured in high-glucose Dulbecco's modified Eagle's medium (DMEM) supplemented with $10 \%$ fetal bovine serum (FBS; Gibco, Grand Island, NY, USA) and incubated in $5 \% \mathrm{CO}_{2}$ at $37^{\circ} \mathrm{C}$.

Real-time-quantitative polymerase chain reaction ( $R T$ - $q P C R)$. Total RNA was prepared using TRIzol reagent (Invitrogen, Carlsbad, CA, USA) according to the manufacturer's instructions. Total RNA $(2 \mu \mathrm{g})$ was used to synthesize the first strand of cDNA. qPCR was performed using an Applied Biosystems 7500 Real-Time PCR system and SYBR ${ }^{\circledR}$ Premix Ex Taq ${ }^{\mathrm{TM}}$ kit (Perfect Real-Time) (Takara Bio Inc., Shiga, Japan). Primers used were as follows: Tumor necrosis factor receptor-associated factor 6 (TRAF6) sense, 5'-CCCGCGCA CTAGAACGAGCAA-3' and antisense, 5'-GCCATGGCCAC ACAGCAGTCA-3'; NF- $\kappa$ B sense, 5'-CGCGCCGCTTAGGA GGGAGA-3' and antisense, 5'-GGGCCATCTGCTGTTGGC AGT-3'; $\beta$-actin sense, 5'-CAATGAAGATCAAGATCATTG CTCCTCC-3' and antisense, 5'-TCAAGAAAGGGTGTAAC GCAACTAAGTC-3'. The expression of $\beta$-actin served as the input reference. The relative mRNA expression of TRAF6 and $\mathrm{NF}-\kappa \mathrm{B}$ were calculated with the comparative threshold cycle (Ct) $\left(2^{-\Delta \Delta \mathrm{Ct}}\right)$ method.

Protein extraction and western blotting. Western blotting was performed as previously described (17). In brief, cell pellets were lysed in a radioimmunoprecipitation assay (RIPA) buffer with $1 \mathrm{mM}$ phenylmethanesulfonyl fluoride (both from Beyotime Institute of Biotechnology, Haimen, China). The cell lysate was centrifuged at $10,000 \times \mathrm{g}$ for $10 \mathrm{~min}$ at $4^{\circ} \mathrm{C}$ and the supernatant was transferred to a microcentrifuge tube. The protein concentration was determined with a Bicinchoninic Acid Protein Assay kit (Beyotime Institute of Biotechnology) and $20 \mu \mathrm{g}$ proteins were loaded/lane. The protein samples were separated on SDS-PAGE at 10-15\%, and electrotransferred onto nitrocellulose membranes (Amersham Pharmacia Biotech, Zurich, Switzerland). The membranes were blocked by $5 \%$ non-fat milk in Tris-buffered saline Tween-20 (TBST; $\mathrm{pH}$ 7.6) for $60 \mathrm{~min}$ at room temperature, followed by incubation in primary antibodies overnight at $4^{\circ} \mathrm{C}$ and secondary antibodies (Thermo Scientific Pierce, Rockford, IL, USA) for $90 \mathrm{~min}$ at room temperature. After the membranes had been washed 3 times, the proteins were detected using electrochemiluminescence (ECL; Amersham Pharmacia Biotech). The antibodies used for western blot analyses were as follows: APRIL, NF- $\kappa$ B and $\beta$-actin (Cell Signaling Technology, Beverly, MA, USA).

Knockdown of APRIL. The short hairpin RNA targeting APRIL mRNA was constructed by GeneChem Biotechnology (Shanghai, China). The shRNA sequences were: 5'-GATCCGCAACCTTCTTCCCTTCTGCTTCAAGA GAGCAGAAGGGAAGAAGGTTGTTTTTGGAAA-3' and 5'-AGCTTTTCCAAAAAACAACCTTCTTCCCTTCTGCT CTCTTGAAGCAGAAGGGAAGAAGGTTGCG-3'; the negative control sequences were: 5'-GATCCGTTCTCCGAA CGTGTCACGTTTCAAGAGAACGTGACACGTTCGGAG AATTTTTTGGAAA-3' and 5'-AGCTTTTCCAAAAAAT TCTCCGAACGTGTCACGTTCTCTTGAAACGTGACAC GTTCGGAGAACG-3'.

Transfection with shRNA was carried out using Lipofectamine $^{\mathrm{TM}} 2000$ according to the manufacturer's instructions (Invitrogen). Cells transfected with APRIL-shRNA were seeded into 6-well culture plates at a density of $1 \times 10^{5}$ cells/well. Cells were allowed to grow for 24,36 and $72 \mathrm{~h}$ and were then harvested for analysis. Insignificant control siRNA was used as a negative control under similar conditions.

Cell viability assay. To analyze the viability of cells treated with JXY and/or APRIL-shRNA, $1 \times 10^{4}$ cells/well were seeded in 96-well plates containing $0.2 \mathrm{ml}$ of medium. After treatment, MTT (5 mg/ml; Sigma, St. Louis, MO, USA) was added to each well (including the control well) and the mixture was incubated at $37^{\circ} \mathrm{C}$ for $4 \mathrm{~h}$. The culture medium was then replaced with an equal volume of dimethyl sulfoxide (Sigma). After shaking at room temperature for $15 \mathrm{~min}$, the 490-nm absorbance (A490) of each well was determined on a microplate reader (Bio-Rad, Hercules, CA, USA). The cell viability was calculated according to the following formula: Cell viability $(\%)=$ A490 (sample)/A490 (control) $x 100$. 

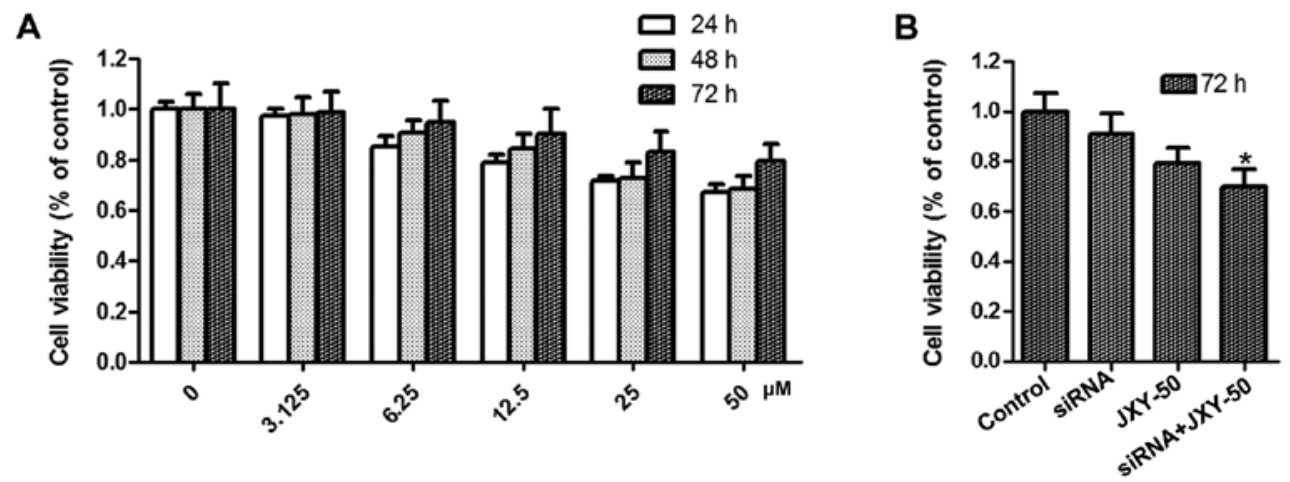

Figure 1. (A) JXY-containing serum at different concentrations inhibits the proliferation of HCC cells in vitro. (B) Comparison of the levels of cell viability after treatment of JXY at the indicated concentrations for $72 \mathrm{~h}$. The viability of the HCC cells was analyzed using methyl thiazolyl tetrazolium (MTT) method. Although JXY treatment had no effect on HCC cell proliferation (P $>0.05$ ), the combination of APRIL knockdown and JXY treatment significantly suppressed cell proliferation in a dose-dependent manner ("P<0.05). Data shown are expressed as the mean \pm standard deviation. JXY, Jiedu Xiaozheng Yin; HCC, hepatocellular carcinoma; APRIL, A proliferation-inducing ligand.

TUNEL assay of cell apoptosis. Cells were seeded onto slides and cultured before being treated with JXY and/or APRIL-shRNA. The cells were then fixed in $4 \%$ paraformaldehyde for $60 \mathrm{~min}$ and permeabilized with $0.1 \%$ Triton X-100 on ice for $2 \mathrm{~min}$. Cell apoptosis was determined using a TUNEL Apoptosis Assay kit (Roche Applied Science, Mannheim, Germany) according to the manufacturer's instructions $48 \mathrm{~h}$ post-transfection. Briefly, TdT-mediated dUTP nick end labeling (TUNEL) reaction mixture was added to the cells for $15 \mathrm{~min}$ and the slides were then rinsed in phosphate-buffered saline (PBS) before being incubated in a humidified chamber at $37^{\circ} \mathrm{C}$ for $60 \mathrm{~min}$ in the dark. Anti-fluorescence quenching solution was added before examination of the cells under a confocal laser-scanning microscope (TCS SP5) at an excitation wavelength of 450-500 $\mathrm{nm}$ and an emission wavelength of 515-565 $\mathrm{nm}$ (green fluorescence) in order to evaluate the proportion of apoptotic cells.

Reporter gene transfection and luciferase activity assay. Cells at $80-90 \%$ confluence growing on a $35-\mathrm{mm}$ dish were co-transfected with the firefly luciferase reporter of $\mathrm{NF}-\kappa \mathrm{B}$ containing a TA promoter $(1 \mu \mathrm{g}, \mathrm{pNF}-\kappa \mathrm{B}-\mathrm{TA}-\mathrm{luc}$; Beyotime Biotechnology, Shanghai, China) along with the Renilla luciferase reporter $(0.1 \mu \mathrm{g}$; Promega Co., Madison, WI, USA) for $6 \mathrm{~h}$ using Lipofectamine ${ }^{\mathrm{TM}} 2000$ according to the manufacturer's instructions (Invitrogen). Some cells were further treated with JXY and/or APRIL shRNA. The luciferase activity was assessed in the cellular extracts using a dual-luciferase reporter gene assay kit (Beyotime Biotechnology. Briefly, the relative fluorescence light unit (RLU) at $560 \mathrm{~nm}$ of the mixture consisting of $50 \mu \mathrm{l}$ total cell lysate and $100 \mu \mathrm{l}$ of the firefly luciferase assay reagent was evaluated using a multimode microplate reader (Tecan Infinite M200; Tecan, Männedorf, Switzerland) for a total period of $10 \mathrm{sec}$. Then, $100 \mu \mathrm{l}$ of Renilla luciferase assay reagent was added into the aforementioned mixture and its fluorescence at $465 \mathrm{~nm}$ was measured. The relative activity of the reporter gene was calculated by dividing the RLU at $560 \mathrm{~nm}$ by that at $465 \mathrm{~nm}$.

Statistical analysis. Data are the results from at least 3 independent experiments. All data are presented as the means \pm SD.
Table I. Synergistic effect of APRIL knockdown or/and JXY treatment on cell cycle distribution in HCC cells.

\begin{tabular}{lccc}
\hline Treatment & G1 $(\%)$ & G2/M $(\%)$ & S $(\%)$ \\
\hline Blank & $42.46 \pm 2.35$ & $38.93 \pm 2.03$ & $18.16 \pm 1.97$ \\
Control & $43.39 \pm 4.18$ & $33.06 \pm 3.17$ & $23.55 \pm 2.52$ \\
shRNA & $49.68 \pm 4.76$ & $37.09 \pm 3.63$ & $13.23 \pm 2.98^{\mathrm{a}}$ \\
Herb & $44.69 \pm 3.89$ & $22.06 \pm 3.53^{\mathrm{a}, \mathrm{b}}$ & $33.25 \pm 3.43$ \\
shRNA + Herb & $59.01 \pm 5.01^{\mathrm{a}, \mathrm{b}}$ & $20.6 \pm 2.99^{\mathrm{a}, \mathrm{b}}$ & $20.39 \pm 3.05$
\end{tabular}

Combination of APRIL knockdown and JXY treatment significantly induced G0/G1 cell cycle arrest. Values are the mean \pm standard deviation; ${ }^{\mathrm{a}} \mathrm{P}<0.05$ vs. the control cells; ${ }^{\mathrm{b}} \mathrm{P}<0.05$ vs. the cells treated with shRNA alone. APRIL, proliferation-inducing ligand; JXY (Herb), Jiedu Xiaozheng Yin; HCC, hepatocellular carcinoma.

Significance was assessed using one-way ANOVA among groups or unpaired t-test for 2 groups. All P-values were two-sided, and the differences were considered significant at a value of $\mathrm{P}<0.05$. All statistical analyses were carried out using SPSS 18.0

\section{Results}

Synergistic inhibitory effect of APRIL knockdown and $J X Y$-containing serum on HCC cell proliferation. To investigate the effect of APRIL knockdown and/or JXY treatment on HCC cell proliferation, an MTT assay was performed in HCC cells following treatment with JXY-containing serum at different concentrations. As shown in Fig. 1, in comparison with the control groups, APRIL knockdown had no effect on HCC cell proliferation while APRIL knockdown significantly enhanced the inhibitory effect of JXY on cell proliferation $(\mathrm{P}<0.05)$. Collectively, these results indicated that the synergistic effect of APRIL knockdown and JXY suppressed HCC cell proliferation.

Synergistic effect of APRIL knockdown and JXY on cell cycle arrest. A DNA cell cycle analysis was performed, in order to 

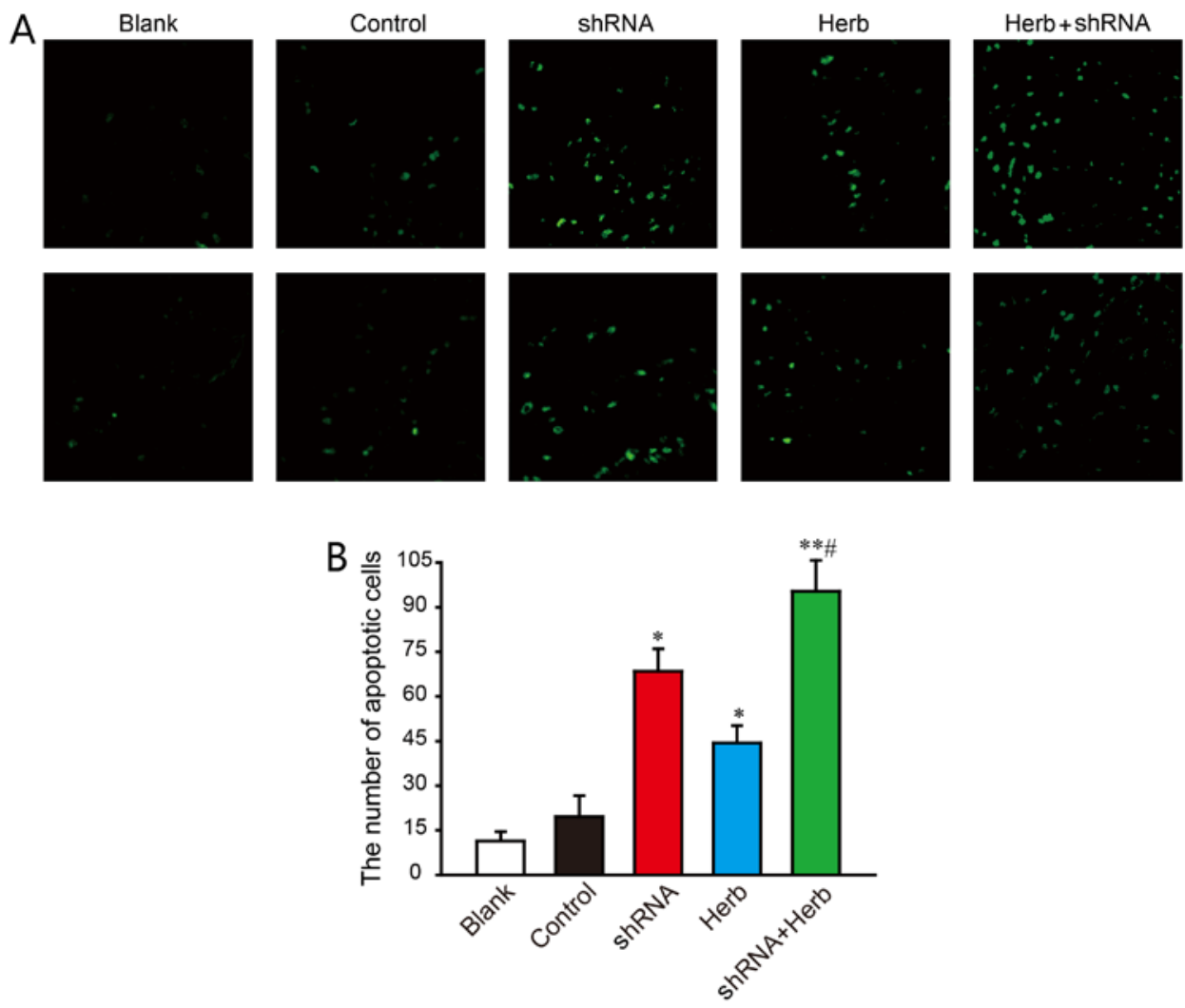

Figure 2. Effect of APRIL knockdown and JXY treatment on HCC cell apoptosis as confirmed by TUNEL assay. (A) The TUNEL assay was carried out by One Step TUNEL Apoptosis Assay kit. The images of TUNEL-positive cells were captured by a fluorescence microscope (magnification, x200). (B) The quantitative result of the TUNEL assay was analyzed. Data are represented as the mean \pm standard deviation; ${ }^{*} \mathrm{P}<0.05$ vs. the control cells; ${ }^{* *} \mathrm{P}<0.05$ vs. the cells treated with JXY (Herb) alone; ${ }^{*} \mathrm{P}<0.05$ vs. the cells treated with shRNA alone. APRIL, A proliferation-inducing ligand; JXY, Jiedu Xiaozheng Yin; HCC, hepatocellular carcinoma; TUNEL, TdT-mediated dUTP nick end labeling.

assess the effects of APRIL knockdown and/or JXY treatment on cell cycle distribution. As shown in Table I, treatment with JXY or APRIL knockdown resulted in an accumulation of cells in the G0/G1 phase of the cell cycle. Notably, the combination of APRIL knockdown and JXY treatment significantly induced G0/G1 cell cycle arrest (shRNA + Herb 59.01 $55.01 \%$ vs. Herb 44.69 $\pm 3.89 \%$; shRNA + Herb $59.01 \pm 5.01 \%$ vs. shRNA 49.68 $\pm 4.76 \%)(\mathrm{P}<0.05)$.

Next, we detected the percentage of cell apoptosis using TUNEL staining. The results showed that both JXY treatment and APRIL knockdown increases the percentage of cell apoptosis ( $\mathrm{P}<0.05$; Fig. 2). In addition, combination of APRIL knockdown and JXY treatment significantly enhanced the number of apoptotic cells ( $\mathrm{P}<0.05$; Fig. 2). Collectively, these results indicated the synergistic effect of APRIL knockdown and JXY treatment on inhibition of HCC cell growth possibly through the induction of G0/G1 cell cycle arrest and the promotion of cell apoptosis.

Synergistic effect of APRIL knockdown and JXY treatment inhibits $N F-\kappa B$ expression. TRAF6 is a unique adaptor protein of the tumor necrosis factor receptor-associated factor family that mediates both tumor necrosis factor receptors and interleukin-1 receptor/Toll-like receptor signaling. TRAF6 plays a very important role in NF- $\kappa \mathrm{B}$ activation $(18,19)$. Recent studies have shown that TRAF6/NF- $\kappa \mathrm{B}$ signaling pathways play an important role in tumorigenesis and invasion $(20,21)$. Next, we explored whether APRIL knockdown and/or JXY treatment could affect TRAF6 and NF- $\mathrm{BB}$ expression. RT-qPCR (Fig. 3) and western blotting (Fig. 4) results showed that APRIL knockdown decreased NF- $\kappa \mathrm{B}$ mRNA and protein expression levels $(\mathrm{P}<0.05)$, but no difference was found in the expression level of TRAF6 (P>0.05). In addition, the expression levels of both TRAF6 and NF- $\mathrm{KB}$ were not altered after JXY treatment. However, the combination of APRIL knockdown and JXY treatment significantly reduced the expression of $\mathrm{NF}-\kappa \mathrm{B}(\mathrm{P}<0.05$; Figs. 3 and 4). These results suggest that the synergistic effect of APRIL knockdown and JXY treatment in the inhibition of $\mathrm{NF}-\kappa \mathrm{B}$ expression was dependent on TRAF6 signaling.

Synergistic effect of APRIL knockdown and JXY treatment decreases the activity of $N F-\kappa B$. A previous study revealed that APRIL could upregulate the activity of nuclear factor $\mathrm{NF}-\kappa \mathrm{B}$ (22). Therefore, we speculated that APRIL knockdown and/or JXY treatment may downregulate the activity of nuclear factor $N F-\kappa B$ directly. Luciferase activity of pNF- $\kappa$ B-TA-Luc that contained multiple copies of the $\mathrm{NF}-\kappa \mathrm{B}$ responsive element was used for monitoring NF- $\kappa \mathrm{B}$ activity (23). As shown in Fig. 5, the result of $\mathrm{NF}-\kappa \mathrm{B}$-controlled luciferase reporter assay revealed that after the knockdown of APRIL, the activity of $\mathrm{NF}-\kappa \mathrm{B}$ was significantly decreased $(\mathrm{P}<0.05)$. In addition, the activity of NF- $\kappa \mathrm{B}$ in the JXY-treated cells was also lower than that in the non-treated cells $(\mathrm{P}<0.05)$. Among the groups, the activity of NF- $\mathrm{NB}$ was the lowest in the combination group 

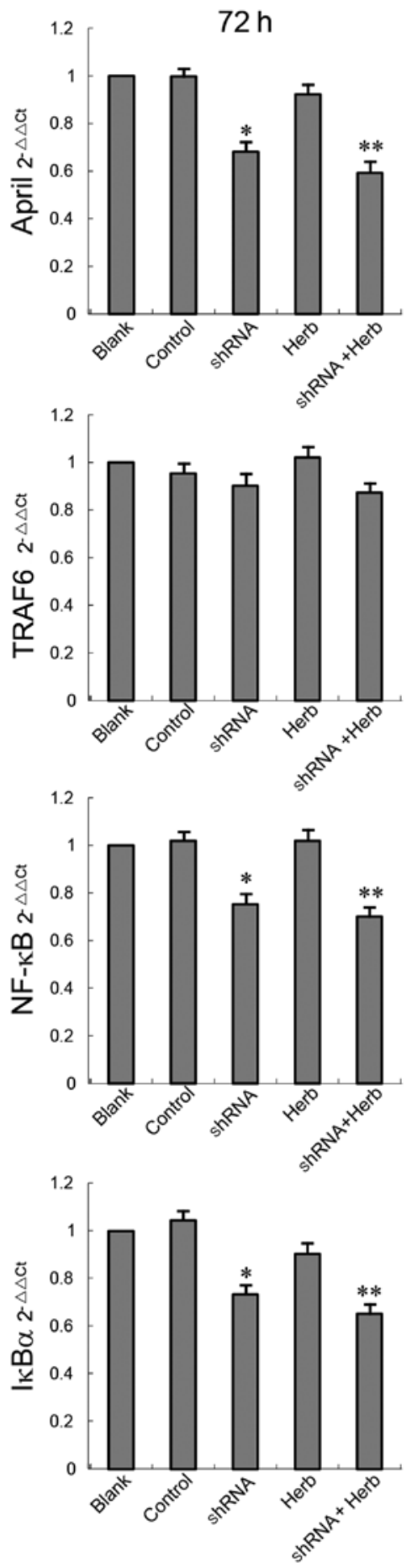

Figure 3. Effect of APRIL knockdown and JXY treatment on mRNA expression levels in HCC cells. The mRNA expression levels of APRIL, TRAF6, $\mathrm{NF}-\kappa \mathrm{B}$ and I $\mathrm{B} \alpha$ were determined by reverse transcription-polymerase chain reaction and quantified by densitometric analysis. Images are representative of 3 independent experiments. The data were normalized to the mean mRNA expression level of the untreated control; ${ }^{*} \mathrm{P}<0.05$ vs. the control cells; ${ }^{* *} \mathrm{P}<0.05$ vs. the cells treated with Herb alone. APRIL, A proliferationinducing ligand; JXY (Herb), Jiedu Xiaozheng Yin; HCC, hepatocellular carcinoma; TRAF6, tumor necrosis factor receptor-associated factor 6 ; NF- $\kappa B$, nuclear factor- $\kappa \mathrm{B}$.

(shRNA + Herb; $\mathrm{P}<0.05$ ). Therefore, the synergistic effect of APRIL knockdown and JXY treatment can trigger cell cycle arrest and cell apoptosis and subsequent suppression of HCC cell proliferation through an NF- $\mathrm{kB}$-related pathway.
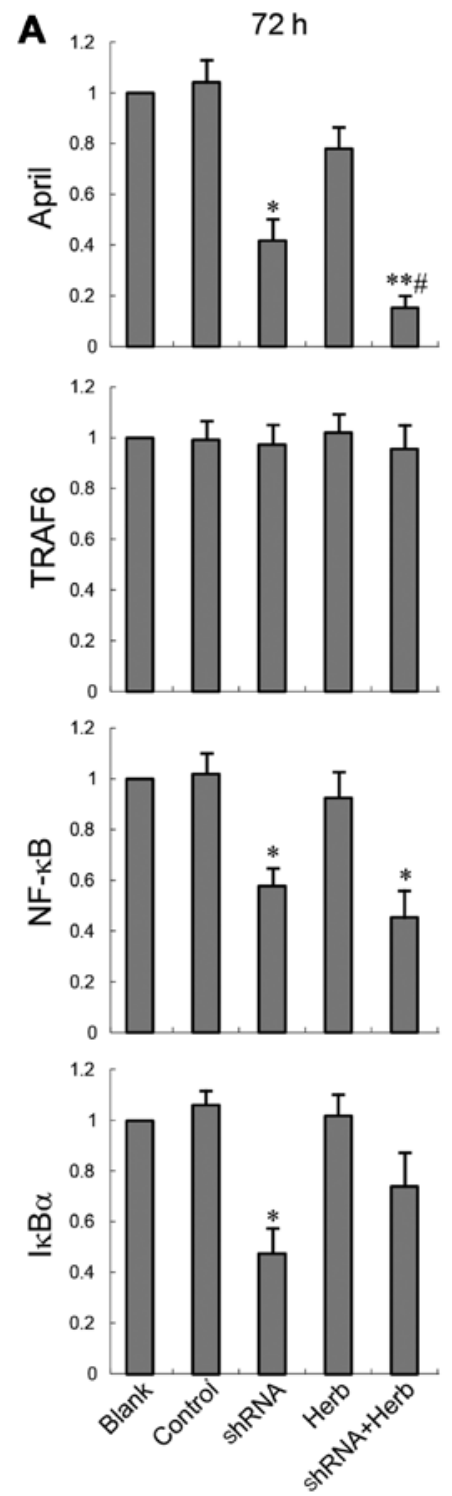

B

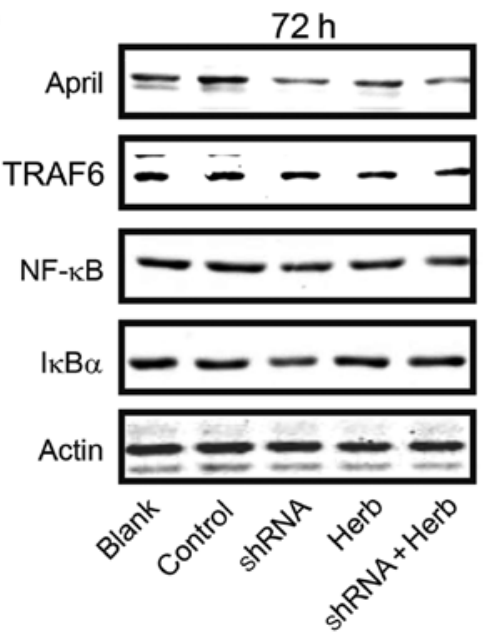

Figure 4. Effect of APRIL knockdown and JXY treatment on protein expression levels in HCC cells. (A) The expression level of all proteins was quantified by densitometric analysis. (B) The expression levels of proteins associated with the $\mathrm{NF}-\kappa \mathrm{B}$ pathway were assessed by western blot analysis. Data shown are expressed as the mean \pm standard deviation of 3 repeated experiments; ${ }^{*} \mathrm{P}<0.05$ vs. the control cells; ${ }^{* *} \mathrm{P}<0.05$ vs. the cells treated with the Herb alone; ${ }^{~} \mathrm{P}<0.05$ vs. the cells treated with shRNA alone. APRIL, A proliferation-inducing ligand; JXY (Herb), Jiedu Xiaozheng Yin; HCC, hepatocellular carcinoma; TRAF6, tumor necrosis factor receptor-associated factor 6 ; NF- $\kappa \mathrm{B}$, nuclear factor- $\kappa \mathrm{B}$. 


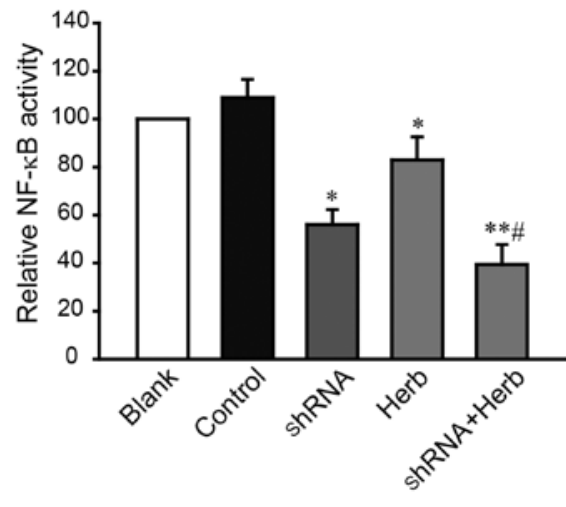

Figure 5. APRIL knockdown and JXY treatment synergistically decrease the activity of NF- $\kappa \mathrm{B}$ in HCC cells. The NF- $\kappa$ B activity in HCC cells was analyzed by an NF-kB reporter luciferase assay. Data shown are from representative experiments where electroporations were performed in triplicate Activation is indicated by the mean \pm standard deviation; ${ }^{*} \mathrm{P}<0.05$ vs. the control cells; ${ }^{* *} \mathrm{P}<0.05$ vs. the cells treated with the Herb alone; ${ }^{*} \mathrm{P}<0.05$ vs. the cells treated with shRNA alone. APRIL, A proliferation-inducing ligand; JXY (Herb), Jiedu Xiaozheng Yin; HCC, hepatocellular carcinoma.

\section{Discussion}

JXY is a Chinese Traditional Medicinal recipe from Chinese herbs exhibiting heat-clearing and detoxification activity. Hedyotis diffusa Willd and Sophora flavescens have been demonstrated to exhibit antiproliferative properties, promote apoptosis and inhibit cell invasion through an NF- $\mathrm{kB}-$ related pathway in a number of cancer cell lines $(24,25)$. In the present study, we demonstrated that APRIL knockdown and JXY treatment synergistically triggered cell cycle arrest and cell apoptosis and subsequent suppression of HCC cell proliferation through an NF-kB-related pathway. Due to its safety profile, JXY is a valuable adjuvant therapy for HCC patients.

APRIL has been implicated in the development of tumors (26). Exogenous APRIL confers a survival advantage to tumor cells from apoptosis $(27,28)$. Thus, APRIL plays a key role in human tumor growth (29). RNA interference (RNAi) has been widely used in the current research of gene therapy for tumors. Recent progress has demonstrated that the siRNA technique is an efficient method for silencing specific genes $(30,31)$. For siRNA to be applied therapeutically, however, several problems need to be solved, such as the short half-life and delivery method of siRNA-mediated silencing. Despite these problems, siRNA remains a potentially promising technique for the treatment of human cancer, particularly given that it can be more selective and less toxic than traditional approaches (32). Furthermore, combination of the siRNA technique with other medications may be a more effective treatment for HCC.

JXY, a TCM polyherbal decoction, is used to treat HCC. In the present study, we evaluated the synergistic effect of APRIL knockdown and JXY treatment on the proliferation of HCC cells. The results showed that transfection with siRNA-APRIL resulted in significant inhibition of APRIL mRNA and protein levels in HepG2 cells. Moreover, APRIL depletion also inhibited cell proliferation and induced G0/G1 cell cycle arrest and apoptosis in HepG2 cells, whereas no inhibitory effect was observed in non-transfected and siRNA control transfected cells. Although the inhibitory effect of cell growth in HepG2 cells was not obvious using JXY treatment alone, the combination of siRNA-APRIL and JXY application had a synergistic inhibitory effect.

Next, we explored the underlying mechanisms. Previous studies revealed that constitutive activation of NF- $\mathrm{KB}$ could play an important role in the regulation of genes involved in tumorigenesis, migration and invasion. In contrast, inhibition of NF- $\mathrm{KB}$ activation suppressed migration and invasion $(33,34)$. TRAF6 has been found to play an important role in tumorigenesis, metastasis and invasion by NF- $\kappa \mathrm{B}$ activation $(35,36)$. Herein, we detected TRAF6 and NF- $\mathrm{BB}$ expression after APRIL knockdown and/or JXY treatment. The results revealed that APRIL knockdown, not JXY treatment, reduced NF- $\kappa \mathrm{B}$ expression. In addition, the combination of APRIL knockdown and JXY treatment significantly reduced NF- $\kappa \mathrm{B}$ expression. However, all of the treatments had no effect on TRAF6 expression. These results suggest that the synergistic effect of APRIL knockdown and JXY treatment on the inhibition of NF- $\kappa B$ expression are not TRAF6-dependent. Furthermore, we found that APRIL knockdown and/or JXY treatment could downregulate the activity of nuclear factor $\mathrm{NF}-\kappa \mathrm{B}$ directly. Collectively, we propose that the synergistic effect of APRIL knockdown and JXY treatment triggers cell cycle arrest and cell apoptosis and subsequent suppression of HCC cell proliferation through the inhibition of the NF- $\kappa B$ signaling pathway.

In conclusion, the present study suggests that APRIL is a feasible RNAi target gene for HCC and the combination of siRNA-APRIL and JXY holds great promise as a novel therapeutic approach for APRIL-positive HCC.

\section{Acknowledgements}

The present study was supported by the University Distinguished Young Research Talent Training Program of Fujian Province, the Fujian Province Natural Science Foundation (nos. 2010J01197 and 2015J01689), the National Natural Science Foundation of China (81202856) and the International Science Joint Project of the Ministry of Science and Technology of China (2008DFA32200).

\section{References}

1. Siegel RL, Miller KD and Jemal A: Cancer statistics, 2016. CA Cancer J Clin 66: 7-30, 2016.

2. Chen W, Zheng R, Baade PD, Zhang S, Zeng H, Bray F, Jemal A, Yu XQ and He J: Cancer statistics in China, 2015. CA Cancer J Clin 66: 115-132, 2016.

3. El-Serag HB: Hepatocellular carcinoma. N Engl J Med 365: 1118-1127, 2011.

4. Hahne M, Kataoka T, Schröter M, Hofmann K, Irmler M, Bodmer JL, Schneider P, Bornand T, Holler N, French LE, et al: APRIL, a new ligand of the tumor necrosis factor family, stimulates tumor cell growth. J Exp Med 188: 1185-1190, 1998.

5. Mohr E, Serre K, Manz RA, Cunningham AF, Khan M, Hardie DL, Bird R and MacLennan IC: Dendritic cells and monocyte/macrophages that create the IL-6/APRIL-rich lymph node microenvironments where plasmablasts mature. J Immunol 182: 2113-2123, 2009.

6. Varfolomeev E, Kischkel F, Martin F, Seshasayee D, Wang H, Lawrence D, Olsson C, Tom L, Erickson S, French D, et al: APRIL-deficient mice have normal immune system development. Mol Cell Biol 24: 997-1006, 2004. 
7. Planelles L, Medema JP, Hahne M and Hardenberg G: The expanding role of APRIL in cancer and immunity. Curr Mol Med 8: 829-844, 2008.

8. Dillon SR, Gross JA, Ansell SM and Novak AJ: An APRIL to remember: Novel TNF ligands as therapeutic targets. Nat Rev Drug Discov 5: 235-246, 2006

9. Ware CF: APRIL and BAFF connect autoimmunity and cancer J Exp Med 192: F35-F38, 2000.

10. Hu M, Zhao M, An C, Yang M, Li Q, Zhang Y, Suetsugu A, Tome Y, Yano S, Fu Y, et al: Real-time imaging of apoptosis induction of human breast cancer cells by the traditional Chinese medicinal herb tubeimu. Anticancer Res 32: 2509-2514, 2012.

11. Park BH, Jung KH, Son MK, Seo JH, Lee HS, Lee JH and Hong SS: Antitumor activity of Pulsatilla koreana extract in anaplastic thyroid cancer via apoptosis and anti-angiogenesis. Mol Med Rep 7: 26-30, 2013

12. Cao Z, Liao L, Chen X, Lan L, Hu H, Liu Z, Chen L, Huang S and $\mathrm{Du} \mathrm{J}$ : Enhancement of antitumor activity of low-dose 5-fluorouracil by combination with Fuzheng-Yiliu granules in hepatoma 22 tumor-bearing mice. Integr Cancer Ther 12: 174-181, 2013.

13. Cao Z, Lin W, Huang Z, Chen X, Zhao J, Zheng L, Ye H, Liu Z, Liao L and Du J: Jiedu Xiaozheng Yin, a Chinese herbal formula, inhibits tumor angiogenesis via downregulation of VEGF-A and VEGFR-2 expression in vivo and in vitro. Oncol Rep 29: 1080-1086, 2013

14. Chen XZ, Cao ZY, Li JN, Hu HX, Zhang YQ, Huang YM, Liu ZZ, Hu D, Liao LM and Du J: Ethyl acetate extract from Jiedu Xiaozheng Yin inhibits the proliferation of human hepatocellular carcinoma cells by suppressing polycomb gene product Bmil and Wnt/ $\beta$-catenin signaling. Oncol Rep 32: 2710-2718, 2014.

15. Cao Z, Lin W, Huang Z, Chen X, Zhao J, Zheng L, Ye H, Liu Z Liao $\mathrm{L}$ and $\mathrm{Du} \mathrm{J}$ : Ethyl acetate extraction from a Chinese herbal formula, Jiedu Xiaozheng Yin, inhibits the proliferation of hepatocellular carcinoma cells via induction of G0/G1 phase arrest in vivo and in vitro. Int J Oncol 42: 202-210, 2013.

16. Chen LW, Lin J, Chen W and Zhang W: Effect of Chinese herbal medicine on patients with primary hepatic carcinoma in III stage during perioperational period: A report of 42 cases. Zhongguo Zhong Xi Yi Jie He Za Zhi 25: 832-834, 2005 (In Chinese)

17. Han T, Jiao F, Hu H, Yuan C, Wang L, Jin ZL, Song WF and Wang LW: EZH2 promotes cell migration and invasion but not alters cell proliferation by suppressing E-cadherin, partly through association with MALAT-1 in pancreatic cancer. Oncotarget 7: 11194-11207, 2016.

18. Zhang X, Zhang J, Zhang L, van Dam $H$ and ten Dijke P: UBE2O negatively regulates TRAF6-mediated NF- $\kappa \mathrm{B}$ activation by inhibiting TRAF6 polyubiquitination. Cell Res 23: 366-377, 2013.

19. Saito S, Murata T, Kanda T, Isomura H, Narita Y, Sugimoto A, Kawashima D and Tsurumi T: Epstein-Barr virus deubiquitinase downregulates TRAF6-mediated NF- $\kappa \mathrm{B}$ signaling during productive replication. J Virol 87: 4060-4070, 2013.

20. Zhong L, Cao F and You Q: Effect of TRAF6 on the biological behavior of human lung adenocarcinoma cell. Tumour Biol 34 231-239, 2013

21. Inoue J, Gohda J, Akiyama T and Semba K: NF-kappaB activation in development and progression of cancer. Cancer Sci 98: $268-274,2007$
22. Zhi X, Tao J, Xiang G, Cao H, Liu Z, Yang K, Lv C and Ni S: APRIL induces cisplatin resistance in gastric cancer cells via activation of the NF- $\kappa \mathrm{B}$ pathway. Cell Physiol Biochem 35: 571-585, 2015.

23. Yuan D, Pan Y, Zhang J and Shao C: Role of nuclear factor-kappaB and P53 in radioadaptive response in Chang live cells. Mutat Res 688: 66-71, 2010.

24. Li Q, Lai Y, Wang C, Xu G, He Z, Shang X, Sun Y, Zhang F, Liu L and Huang H: Matrine inhibits the proliferation, invasion and migration of castration-resistant prostate cancer cells through regulation of the NF- $\mathrm{KB}$ signaling pathway. Oncol Rep 35: 375-381, 2016.

25. Chen Y, Lin Y, Li Y and Li C: Total flavonoids of Hedyotis diffusa Willd inhibit inflammatory responses in LPS-activated macrophages via suppression of the NF- $\kappa$ B and MAPK signaling pathways. Exp Ther Med 11: 1116-1122, 2016.

26. Mackay F and Tangye SG: The role of the BAFF/APRIL system in B cell homeostasis and lymphoid cancers. Curr Opin Pharmacol 4: 347-354, 2004.

27. Klein B, Tarte K, Jourdan M, Mathouk K, Moreaux J, Jourdan E, Legouffe E, De Vos J and Rossi JF: Survival and proliferation factors of normal and malignant plasma cells. Int J Hematol 78: 106-113, 2003.

28. Moreaux J, Legouffe E, Jourdan E, Quittet P, Rème T, Lugagne C, Moine P, Rossi JF, Klein B and Tarte K: BAFF and APRIL protect myeloma cells from apoptosis induced by interleukin 6 deprivation and dexamethasone. Blood 103: 3148-3157, 2004.

29. Moreaux J, Veyrune JL, De Vos J and Klein B: APRIL is overexpressed in cancer: Link with tumor progression. BMC Cancer 9: 83, 2009.

30. Wang J, Ding W, Sun B, Jing R, Huang H, Shi G and Wang H: Targeting of colorectal cancer growth, metastasis, and anti-apoptosis in BALB/c nude mice via APRIL siRNA. Mol Cell Biochem 363: 1-10, 2012.

31. Jiao F, Hu H, Yuan C, Wang L, Jiang W, Jin Z, Guo Z and Wang L: Elevated expression level of long noncoding RNA MALAT-1 facilitates cell growth, migration and invasion in pancreatic cancer. Oncol Rep 32: 2485-2492, 2014.

32. Wang F, Chen L, Ni H, Wang G, Ding W, Cong H, Ju S, Yang S and Wang H: APRIL depletion induces cell cycle arrest and apoptosis through blocking TGF- $\beta 1 /$ ERK signaling pathway in human colorectal cancer cells. Mol Cell Biochem 383: 179-189, 2013.

33. Tao T, Cheng C, Ji Y, Xu G, Zhang J, Zhang L and Shen A: Numbl inhibits glioma cell migration and invasion by suppressing TRAF5-mediated NF- $\mathrm{KB}$ activation. Mol Biol Cell 23: 2635-2644, 2012

34. Sarkar D, Park ES, Emdad L, Lee SG, Su ZZ and Fisher PB Molecular basis of nuclear factor-kappaB activation by astrocyte elevated gene-1. Cancer Res 68: 1478-1484, 2008.

35. Starczynowski DT, Lockwood WW, Deléhouzée S, Chari R, Wegrzyn J, Fuller M, Tsao MS, Lam S, Gazdar AF, Lam WL, et al: TRAF6 is an amplified oncogene bridging the RAS and NF- $\kappa \mathrm{B}$ pathways in human lung cancer. J Clin Invest 121: 4095-4105, 2011.

36. Ramachandran C, Rodriguez S, Ramachandran R, Raveendran Nair PK, Fonseca H, Khatib Z, Escalon E and Melnick SJ: Expression profiles of apoptotic genes induced by curcumin in human breast cancer and mammary epithelial cell lines. Anticancer Res 25: 3293-3302, 2005. 